\title{
Covering a Square by Small Perimeter Rectangles*
}

\author{
N. Alon ${ }^{1, * *}$ and D. J. Kleitman ${ }^{2, * * *}$ \\ 'Massachusetts Institute of Technology, Cambridge, MA and Bell Communications Research, \\ Morristown, NJ 07960 \\ ${ }^{2}$ Massachusetts Institute of Technology, Department of Applied Mathematics, Cambridge, MA 02139
}

\begin{abstract}
We show that if the unit square is covered by $n$ rectangles, then at least one must have perimeter at least $4(2 m+1) /(n+m(m+1))$, where $m$ is the largest integer whose square is at most $n$. This result is exact for $n$ of the form $m(m+1)$ (or $\left.m^{2}\right)$.
\end{abstract}

\section{Introduction}

In this note, we address the following problem, suggested by L. Hurwicz [1]. How can we partition the unit square, $U$, into a given number, $n$, of rectangles (all having edges parallel to those of the square), so as to minimize the largest of their perimeters? The same question has been raised when the rectangles are only required to cover the square, with overlap allowed.

These problems arose from a model of a communication problem, in which $n$ is the number of potential messages, and the perimeter of the associated rectangle is a measure of the uncertainty of successful communication of that message.

We will obtain the same lower bounds for the partition and covering problems for the largest perimeter. In the following discussion we will denote the smallest largest perimeter for given $n$ for the partition problem as $p(n)$.

It is easy to see that $p(n)$ obeys

$$
p(n) \geq 4 / \sqrt{n} \text {, }
$$

* Research supported in part by NSF under contract DMS-8406100.

**Supported in part by the Weizmann Fellowship for Scientific Research.

${ }^{* * *}$ Supported in part by the University of Minnesota under the Ordway Endowment. 
with equality if and only if $n$ is a perfect square, say $m^{2}$. For, the largest rectangle must have area at least $1 / n$ and hence perimeter at least as given; and equality can only hold if all rectangles have area exactly $1 / n$ and are squares, which is only feasible if $n$ is a perfect square.

Here we will improve this trivial lower bound for all non-square $n$. In particular we obtain an exact expression for $n$ of the form $m(m+1)$ with integer $m$, namely,

$$
p(m(m+1))=2 / m+2 /(m+1) .
$$

We also establish an upper bound for $p(n)$ which exceeds our lower bound by at most $\left(\frac{1}{4}+o(1)\right) n^{-3 / 2}$.

In the next section we obtain a linear programming problem whose solution provides a lower bound for $p(n)$. We show this by integrating certain functions over the boundary of the square. In Section 3 we solve the linear program to obtain our lower bound for $p(n)$. In Section 4 we apply these results to the covering problem. We then describe the construction, which has been conjectured to be optimal (by Hurwicz). We conclude with some additional remarks.

\section{The Linear Inequalities}

Let $U$ be the unit square, $U=((x, y): 0 \leq x, y \leq 1)$, and let $P$ be a partition of $U$ into (or covering of $U$ by) rectangles $R_{1}, \ldots, R_{n}$, each having edges parallel to the axes. For each $i$ here let $A_{i}$ denote the area of the rectangle $R_{i}$ and let $p_{i}$ denote its perimeter, with $p$ the largest value of the $p_{t}$ s.

For $0 \leq z<1$, we say that $z$ sees $R_{i}$ if the line $x=z$ intersects $R_{i}$, while for $1 \leq z \leq 2, z$ sees $R$, when the line $y=z-1$ intersects $R_{j}$.

We define $\mu_{k}$, for integral $k$, to be the (one-dimensional Lebesgue) measure of the set of values of $z$ for which $z$ sees exactly $k$ rectangles. Clearly the function $\mu_{k}$ obeys the conditions, $\mu_{k} \geq 0$, and also

$$
\sum_{k=1}^{n} \mu_{k}=2 \text {. }
$$

We will now prove the following two statements:

Proposition 2.1. With the given definitions we have, both for partitioning and covering,

$$
\sum_{k=1}^{n} k \mu_{k}=\frac{1}{2} \sum_{i=1}^{n} p_{i} \leq \frac{n p}{2} .
$$

Proposition 2.2. With these same definitions we have, again for both problems,

$$
\sum_{k=1}^{n} \frac{\mu_{k}}{k} \leq \sum_{i=1}^{n} \frac{p_{i} A_{i}}{2} \leq \frac{\lambda p}{2}
$$

where $\lambda$ is the sum of the areas of all the rectangles. 
It immediately follows from Eq. (2.1) and these two statements that the smallest value of the parameter $p$ that, with any set of $\mu_{k}$, obeys

$$
\begin{aligned}
& \mu_{k} \geq 0 \quad \text { for all } k \\
& \sum_{k=1}^{n} \mu_{i}=2 \\
& p \geq \frac{2}{n} \sum_{k=1}^{n} k \mu_{k}, \\
& p \geq 2 \sum_{k=1}^{n} \frac{\mu_{k}}{k}
\end{aligned}
$$

must provide a lower bound for $p=p(n)$ here, since $p$ must in fact obey all these. This is then a linear program, and our lower bound will be the minimum $p$ solution for it.

We now prove the two statements here.

Proof of Proposition 2.1. We define the function $f(z)$ to be the number of rectangles seen by $z$, which is the sum over each rectangle of 1 if the rectangle is seen by $z$. The integral of $f(z)$ from 0 to 2 will then contain a contribution of $p_{1} / 2$ from the $i$ th rectangle. But it is also the sum of $k \mu_{k}$, from our definitions.

Proof of Proposition 2.2. We define the function $g(z)$ for $z<1$ to be the sum of the squares of the lengths of the rectangles seen by $z$, where by length we mean size measured along the line $x=z$. If we denote these rectangles by $Z_{1}, \ldots, Z_{k}$, and their lengths by $a_{1}, \ldots, a_{k}$, respectively, then we have $g(z)=\sum a_{i}^{2}$. (Note that for the partitioning problem these as will sum to one, while in the covering problem they will sum to at least one.)

For $z \geq 1$ we similarly define $g(z)$ to be the sum of the squares of the widths of the rectangles seen by $z$.

We obtain our inequality by evaluating the integral, $J$, of $g(z)$ from 0 to 2 .

The contribution from the rectangle $R_{i}$, whose length is $l$ and width is $w$, will be $w l^{2}+l w^{2}$ which is $l w(l+w)$ or $\frac{1}{2} A_{i} p_{i}$. We therefore have

$$
J=\sum \frac{p_{i} A_{i}}{2} \leq \frac{1}{2} p \sum A_{i}=\frac{\lambda p}{2} \text {. }
$$

On the other hand, when $z$ sees $k$ rectangles, then $g(z) \geq 1 / k$. For, by the convexity of the square, we have, $g(z)=\sum a_{i}^{2} \geq\left(\sum a_{i}\right)^{2} / k \geq 1 / k$, where the sum has $k$ terms. Thus we have $J \geq \sum \mu_{k} / k$.

\section{The Lower Bound}

We may extract a lower bound for the partition problem from the linear program just described (with $\lambda=1$ ) by producing a feasible point for the dual program. In 
fact, it is easy to solve the dual problem here, since it involves only three variables, two of which are easily eliminated. The resulting solution then gives the best possible bound that can be extracted from the inequalities defining the program. We now state our results.

Proposition 3.1. If $n$ is of the form $m(m+1)$ then $p(n)$ is $2 / m+2 /(m+1)$.

Proposition 3.2. In general, $p(n)$ must be at least $4(2 m+1) /(n+m(m+1))$, where $m$ is the greatest integer whose square is at most $n$.

Proposition 3.1 includes the fact that the regular partition of the square into $m(m+1)$ congruent rectangles of size $1 / m$ by $1 /(m+1)$ realizes the lower bound that we derive.

Proofs. If we let $r, s$, and $t$ be the dual variables associated, respectively, with the constraints of Eq. (2.1), Proposition 2.1, and Proposition 2.2, we obtain as dual program:

$$
\begin{aligned}
& \text { Maximize } 2 r, \text { subject to } \\
& s \geq 0, \quad t \geq 0, \\
& s+t \leq 1, \\
& \text { and for all integer } k \text { with } k \leq n, \\
& r \leq \frac{2 t}{k}+\frac{2 k s}{n} .
\end{aligned}
$$

It is obvious that the maximum here will occur when $s+t=1$, and will occur at the intersection of some of the constraints (3.1). If we set $s=1-t$, and examine the values of $t$ for which the $k$ constraint intersects the $k+1$ constraint, we find that these increase with $k$, while the slopes of these constraints as a function of $t$ decrease with $k$. It then follows that these consecutive intersection points obey all the constraints, so that the value of $2 r$ according to inequalities (3.1) that has the largest $r$ value among them will provide a lower bound to any primal solution, and that will be the best bound that can be obtained from these inequalities.

The $k, k+1$ intersection referred to here occurs when we have

$$
(1-t) \frac{k}{n}+\frac{t}{k}=\frac{(1-t)(k+1)}{n}+\frac{t}{(k+1)},
$$

or for $t$ and $2 r$ given by

$$
\begin{aligned}
& k(k+1)(1-t)=t n, \\
& t=1-\frac{n}{(n+k(k+1))}, \\
& 2 r=\frac{4(2 k+1)}{(n+k(k+1))} .
\end{aligned}
$$


It is obvious geometrically that the bound is maximized when $k^{2} \leq n$ and $(k+1)^{2} \geq n$, since that is the place at which the slope of the lower envelope of these constraints changes sign.

We note that, as $n$ varies from $m^{2}$ to $m(m+1)$ and to $(m+1)^{2}$, the bound given by Proposition 3.2 interpolates between what are exact bounds at these three points, according to a formula that can be written as $a /(n+b)$.

\section{Lower Bounds for the Covering Problem}

We will now show that the lower bounds given in Propositions 3.1 and 3.2 for the partition problem apply as well to the more general covering problem. We do this by replacing the covering by rectangles problem by a partition-into-polyominoes problem, and showing that the results of the last two sections apply as well when the rectangles are replaced by such polyominoes.

A polyomino $L$ is a disjoint union of one or more planar regions with rectilinear axis-parallel sides that lies inside the unit square $U$. Note that $L$ is not necessarily connected. For $0 \leq z<1$, we say that $z$ sees $L$ if the line $x=z$ intersects $L$, and we let $a(L, z)$ denote the total length of this intersection (i.e., the sum of lengths of the intervals in $L \cap\{(x, y): x=z, 0 \leq y \leq 1\}$. Similarly, for $1 \leq z \leq 2$, we say that $z$ sees $L$ if the line $y=z-1$ intersects $L$, and we let $a(L, z)$ denote the total length of this intersection. The width of $L, w(L)$ is the (one-dimensional Lebesgue) measure of the set of values $z, 0 \leq z<1$, that sees $L$. The length of $L, l(L)$ is the measure of the set of values $z, 1 \leq z \leq 2$, that sees $L$.

Notice that

$$
a(L, z) \leq l(L) \text { for all } 0 \leq z<1
$$

and

$$
a(L, z) \leq w(L) \text { for all } 1 \leq z \leq 2
$$
then

Notice also that if $p(L)$ is the perimeter of $L$ and $q(L)=2 l(L)+2 w(L)$

$$
q(L) \leq p(L)
$$

and equality holds iff the intersection of $L$ with every vertical or horizontal line is connected (or empty).

Suppose, now, that the unit square $U$ is partitioned into polyominoes $L_{1}, \ldots, L_{n}$. For each $i$, let $A_{i}$ denote the area of the polyomino $L_{i}$, and put $q_{i}=q\left(L_{i}\right)$. Define $\mu_{k}$, for integral $k$, as in Section 2. Clearly (2.1) holds. Let $q=\max \left(q_{i}: 1 \leq i \leq n\right)$. Imitating the proof of Proposition 2.1 we conclude that

$$
\sum_{k=1}^{n} k \mu_{k}=\frac{1}{2} \sum_{i=1}^{n} q_{i} \leq \frac{n q}{2} .
$$

The next proposition is the analogue of Proposition 2.2 for polyominoes. 
Proposition 4.1. For the partitioning to polyominoes problem

$$
\sum_{k=1}^{n} \frac{\mu_{k}}{k} \leq \sum_{i=1}^{n} \frac{q_{i} A_{i}}{2} \leq \frac{q}{2}
$$

Proof. Define, for $0 \leq z \leq 2$.

$$
g(z)=\sum\left\{a^{2}\left(L_{i}, z\right): L_{i} \text { is seen from } z\right\}
$$

Let $J$ be the integral of $g$ from 0 to 2. By the convexity of the square we obtain, as in the proof of Proposition 2.2, that

$$
J \geq \sum_{k=1}^{n} \frac{\mu_{k}}{k} .
$$

On the other hand, we claim that the contribution of $L_{i}$ to $J$ is at most $\frac{1}{2} q_{i} A_{i}$. Indeed, by inequalities (4.1) and (4.2)

$$
\begin{aligned}
\int_{z=0}^{1} a^{2}\left(L_{i}, z\right)+\int_{1}^{2} a^{2}\left(L_{i}, z\right) & \leq \int_{z=0}^{1} l\left(L_{i}\right) a\left(L_{i}, z\right)+\int_{z=1}^{2} w\left(L_{i}\right) a\left(L_{i}, z\right) \\
& =\left(l\left(L_{i}\right)+w\left(L_{i}\right)\right) A_{i}=\frac{1}{2} q_{i} A_{i} .
\end{aligned}
$$

Hence $J \leq \sum_{i=1}^{n} q_{i} A_{i} / 2 \leq q / 2$, and Proposition 4.1 follows.

We thus conclude that the lower bound for $p(n)$ given by Proposition 3.2 holds also for $q$, and, in view of inequality (4.3), we obtain,

Theorem 4.2. In any partitioning of the unit square into $n$ polyominoes $L_{1}, \ldots, L_{n}$, if $q=\max q\left(L_{1}\right)$ and $p$ is the maximal perimeter of the $L_{i} s$ then

$$
p \geq q \geq \frac{4(2 m+1)}{(n+m(m+1))}
$$

where $m$ is the greatest integer with $m^{2} \leq n$. This is exact for $n=m^{2}$ or $n=m(m+1)$.

Corollary 4.3. The lower bound given by $p(n) \geq 4(2 m+1) /(n+m(m+1))$, with $m$ the greatest integer with $m^{2} \leq n$; which is exact for $n=m^{2}$ or $n=m(m+1)$; holds for any covering of the unit square by axis parallel rectangles.

Proof. Given a covering of $U$ by $n$ rectangles $R_{1}, R_{2}, \ldots, R_{n}$, let $L_{i}$ be the polyomino $R_{i}-\left(R_{i+1} \cup \cdots \cup R_{n}\right)$. Since $L_{i}$ is a subset of $R_{i}, q\left(L_{i}\right)$ is at most the perimeter of $R_{i}$. Since $L_{1}, \ldots, L_{n}$ form a partition of $U$ into polyominoes the result follows from Theorem 4.2 . 


\section{Upper Bound Constructions and Remarks}

When $n=m^{2}$ or $n=m(m+1)$, partitions of the unit square into $n$ rectangles immediately suggest themselves. For other values of $m$, there is no regular partition, and the closest we can come to one is to have two kinds of rectangles, with, for example, some values of $z$, with $z<1$, seeing $m$ rectangles of one kind, and others seeing $m+1$ rectangles of the other kind.

Specifically, when $n=m(m+1)$ we can have $m$ rows with $m+1$ congruent rectangles in each; when $n=m(m+1)-j$, for $j<m$, we instead have only $m$ rectangles in $j$ of the rows, and make all rectangles in the same kind of row identical. The dimensions of the two kinds of rectangles can then be adjusted so that all perimeters are the same. Similarly, when $n=m(m+1)+j$, for $j \leq m$ we may have $m+1$ rectangles in each of $j$ columns and $m$ rectangles in the $m+1-j$ other columns.

These constructions seem to be best possible, and have been conjectured to be so by Hurwicz, for covering as well as partitioning. That they are so has been proven for small values of $n$.

The upper bound on minimum maximum perimeter that follows from these constructions agrees with the lower bounds for $n=m^{2}, m(m+1)$, and $(m+1)^{2}$, and curiously, provides a linear interpolation between these values for other $n$.

Thus for $n$ between $m^{2}$ and $m(m+1)$, the upper bound we get here is $4\left(3 m^{2}+2 m-n\right) /\left(2 m^{2}(m+1)\right)$; while for $n$ between $m(m+1)$ and $(m+1)^{2}$ we get an upper bound of $4((3 m+1)(m+1)-n) /\left(2(m+1)^{2} m\right)$.

We conclude with the following remarks:

1. Hurwicz conjectured that the upper bound given is actually best even if one is allowed to tilt the rectangles. It is dubious that tilting does any good here, and it would be nice to be able to prove so.

2. The difference between upper and lower bounds here fluctuates between zero and an upper bound that behaves as $n^{-3 / 2} / 4(1+o(1))$.

3. It would be nice to be able to handle values of $n$ that are not squares or of the form $m(m+1)$, as well as similar problems in higher dimensions.

\section{Reference}

1. L. Hurwicz, private communication.

Received Mav 31, 1985, and in revised form July 16, 1985. 